03

\title{
Оценка интенсивности электромагнитного излучения шаровой молнии
}

\author{
() А.И. Григорьев, С.О. Ширяева \\ Ярославский государственный университет им. П.Г. Демидова, \\ 150000 Ярославль, Россия \\ e-mail: grig@uniyar.ac.ru
}

(Поступило в Редакцию 23 февраля 2018 г.)

На основе списка свойств шаровой молнии, составленного по описаниям ее существования в естественных условиях, проведена оценка по порядку величины максимальной интенсивности ее электромагнитного микроволнового излучения. Физическим механизмом генерации микроволнового излучения шаровой молнии может являться движение заряженных частиц ее вещества по криволинейным траекториям внутри занимаемого ею объема.

DOI: $10.21883 / J T F .2019 .01 .46956 .84-18$

Проблема исследования происхождения, строения и истолкования наблюдаемых свойств шаровой молнии (ШМ) появилась еще в античные времена, когда знание находилось в стадии накопления сведений об окружающем мире и их философском осмыслении. В середине XIX в., когда уже сформировался „институт“ науки как социального явления, систематизирующего старые и производящего новые знания, в одной из первых монографий по атмосферному электричеству [1] уже содержалась большая часть фактических сведений о ШМ, и были сформулированы (пусть и в неявной форме) основные загадки, связанные с ШМ. Сам автор [1] Франсуа Араго (почетный иностранный член Петербургской Академии Наук) назвал ШМ „самым необъяснимым физическим явлением“. В таком статусе она пребывает и по нынешний день. Конечно, за прошедшие годы кое-что сделано, но сделанное касается в основном уточнения списка наблюдаемых свойств ШМ и сводится к изданию двух десятков обзоров и монографий на эту тему (см., например, [2-12] и указанную там литературу).

C конца XX века стали регулярно проводиться международные симпозиумы [13] и национальные конференции [14] по проблеме ШМ, которые, однако, пока не привели к существенному прогрессу в понимании этого феномена. Хотя определенные сдвиги, безусловно, имеются.

Основной метод познания в этой сфере знания это сбор информации у населения о встречах с ШМ в естественных условиях и моделирование. Количество предложенных теоретических и экспериментальных моделей исчисляется сотнями [2-14]. Привязка большинства моделей к конкретным наблюдаемым свойствам ШМ чисто условная. Как правило, моделируется просто нечто приблизительно сферическое и светящееся. В столь широкие рамки попадает и люминесценция азота под действием ультрафиолетового излучения, и разлетающиеся капли горящего металла от места контакта дуги трамвая с проводом или от места контакта струи плазмы капиллярного разряда с металлической фольгой [15-17] и многое другое.

Отрадным явлением в плане экспериментального моделирования ШМ в последние десятилетия является создание и изучение современными средствами так называемых долгоживущих плазменных образований (ДПО) [18-21]. ДПО чисто внешне напоминают ШМ, хотя не обладают характерным набором свойств реальной ШМ, из которых большая длительность существования и относительно большой энергетический запас являются определяющими $[1,2,5,6,11]$. ДПО, получаемые как в маломощных $[16,18,19,21]$, так и в мощных [20] разрядах, живут $\sim 1 \mathrm{~s}$. Энергия ДПО измеряется единицами джоулей. В реальности ДПО не может даже газетный лист прожечь (см., например, рисунок, где приведена фотография ДПО, полученная Г.Д. Шабановым [22]).

Об энергиях реальных ШМ говорить сложно: слишком большой разброс и низкая достоверность разных оценок. Однако, согласно наблюдениям, энергии ШМ достаточно, чтобы светить с интенсивностью в течение

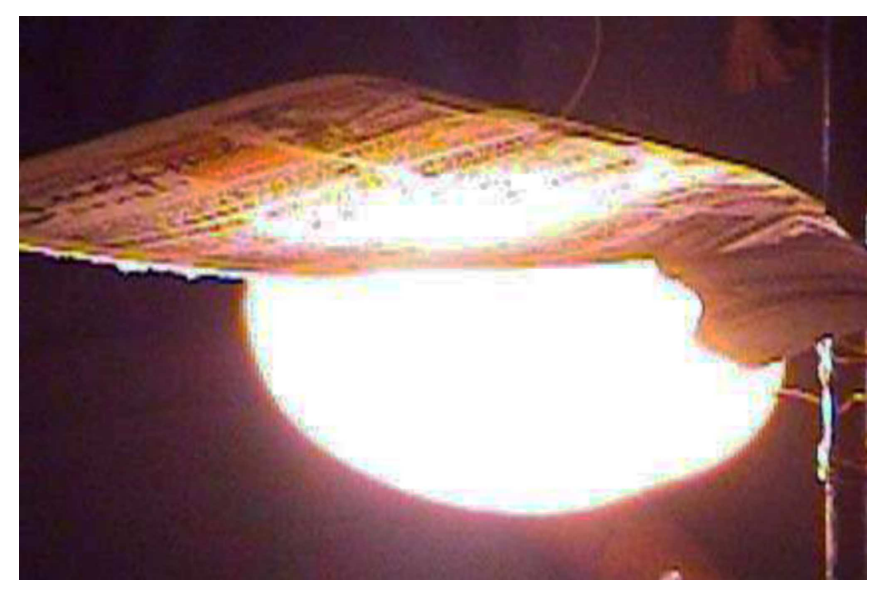

Контакт ДПО с газетой. Фотография из работы [22] Г.Д. Шабанова и др. 
десятков секунд, или же поджечь дом или стог сена во время дождя $[1,2,5,6,11,12]$. Если не принимать во внимание оценки энергии ШМ авторами различных теоретических моделей, то, как правило, энергию ШМ оценивают по разрушениям, произведенным при ее взрыве. Но вместе с тем взрываются не все ШМ (около четверти [12]), большинство же просто тихо гаснет или уходит из поля зрения наблюдателя.

Тем не менее представляется, что создание и продолжающееся изучение ДПО является заметным шагом вперед по сравнению с созерцательным сбором данных случайных наблюдений.

В сфере теоретического моделирования последние несколько десятков лет также имеются позитивные сдвиги: появилась продуктивная тенденция: моделировать не все явление сразу, а лишь отдельные свойства ШМ [23-28], исходя лишь из общефизических представлений и не задаваясь вопросами о происхождении ШМ и ее строении, но опираясь, однако, на перечень наблюдаемых ее физически обусловленных свойств.

Так, в [23] исходя из факта, что вещество ШМ не смешивается с окружающим воздухом при ее движении, делается вывод о наличии четко очерченной границы раздела: вещество ШМ-воздух, и исследуется устойчивость этой границы по отношению к полю силы тяжести. В [24,26] разными авторами предприняты попытки исследования особенностей прохождения ШМ сквозь стекло. В [25] оценивается время жизни ШМ в предположении, что основным источником потерь ею энергии является световое излучение. В [27] для ШМ, плавающей в воздухе в суперпозиции гравитационного и приземного электростатического полей, из соображений ограниченности напряженности электростатического поля у поверхности ШМ пробойной величиной (напряженность электрического поля у поверхности ШМ, при которой зажигается коронный разряд) находится плотность электростатической энергии ее нескомпенсированного заряда. Полученная оценка составила порядка тысячной доли джоуля на кубический сантиметр. В [28] для ШМ, плавающей в воздухе в суперпозиции гравитационного и приземного электрического полей, показано, что при фиксированных прочих физических характеристиках, радиус ШМ, отношение плотностей вещества ШМ и окружающей среды, а также величина коэффициента поверхностного натяжения границы раздела не могут превышать определенных, физически разумных (никакой фантастики) значений.

В настоящей работе предполагается рассмотреть давно известное и экзотическое свойство ШМ, а именно ее способность излучать электромагнитные волны и заставлять светиться не включенные в сеть электролампочки. О такой способности ШМ упоминалось в монографии Бранда [2], писали о ней и авторы более поздних обзоров и книг [3-6,8-12]. Это свойство ШМ интересно тем, что позволяет сделать определенные выводы о ее устройстве. Попробуем смоделировать это свойство, исходя из наиболее общих физических положений.
1. Согласно свидетельствам очевидцев, при пролете ШМ рядом с выключенными из электросети лампочками, последние начинают светиться и погасают при удалении ШМ. В некоторых случаях очевидцы говорят лишь о слабом свечении нити накаливания.

Исходя из того, что наблюдаемое явление обусловлено физически, следует предположить, что ШМ вызывает в нити накаливания лампочки индукционные токи, разогревающие спираль. А значит, ШМ должна либо иметь весьма сильное собственное постоянное магнитное поле, либо излучать электромагнитные волны. На наличие у ШМ постоянного магнитного поля указывает лишь одно описание (в котором ШМ намагнитила хирургические инструменты) в [3]. В нескольких тысячах собранных описаний наблюдений ШМ в естественных условиях такой способности ШМ больше не отмечалось. Остается уповать на электромагнитное излучение ШМ.

В силу известного закона Фарадея

$$
\varepsilon_{\text {ind }}=-\frac{\partial \Phi}{\partial t}
$$

при изменении во времени $\Phi-$ потока магнитного поля через поперечное сечение нагревательной спирали лампочки, в спирали индуцируется ЭДС $\varepsilon_{\text {ind }}$, которая вызывает ток, разогревающий спираль и заставляющий ее светиться.

Нижеследующие оценки проведем по порядку величины, моделируя из соображений простоты нить накаливания прямым соленоидом.

2. Начнем с простого металлического кольца радиуca $r$, через которое перпендикулярно плоскости кольца проходит поток $\Phi$ магнитного поля напряженностью

$$
H(t)=H_{0} \cos \left[-\omega\left(t-\frac{x}{c}\right)\right],
$$

изменяющейся во времени с частотой $\omega$. Здесь $c-$ скорость света в вакууме. Умножая площадь кольца $\pi r^{2}$ на величину вектора магнитной индукции $B(t) \equiv \mu_{0} \mu H(t)$, найдем, что изменение потока магнитного поля, проходящего ежесекундно сквозь кольцо, будет равно

$$
\frac{\partial \Phi}{\partial t}=\pi r^{2} \frac{\partial B(t)}{\partial t} \equiv \mu_{0} \mu \pi r^{2} \omega H_{0} \sin \left[-\omega\left(t-\frac{x}{c}\right)\right],
$$

где $\mu$ - магнитная проницаемость среды (для лампочки это магнитная проницаемость газа, которым заполнена лампочка, т.е. примерно единица), $\mu_{0}-$ магнитная постоянная.

В силу закона Фарадея, для получим выражение

$$
\varepsilon_{\text {ind }}=-\frac{\partial \Phi}{\partial t} \equiv-\mu_{0} \mu \pi r^{2} \omega H_{0} \sin \left[-\omega\left(t-\frac{x}{c}\right)\right] .
$$

3. Перейдем к электролампочке. Нить накаливания лампочки будем моделировать прямым соленоидом, длина которого $l$ много больше его радиуса $r$ (т.е. $l \gg r$ ). В реальных лампочках длина спирали (соленоида) $l$ 
по порядку величины обычно измеряется сантиметрами, а радиус спирали (соленоида) $r$ порядка долей миллиметра (около трех-четырех десятых миллиметра). Сама проволочка (обычно вольфрамовая) имеет толщину $\sim 0.15 \mathrm{~mm}$.

В итоге ЭДС индукции нити лампочки будет в $n l$ раз больше, чем в одиночном кольце:

$$
\varepsilon_{\text {ind }}=-n l \cdot \mu \mu_{0} \pi r^{2} \omega H_{0} \sin \left[-\omega\left(t-\frac{x}{c}\right)\right],
$$

$n-$ количество витков на единицу длины.

Для оценки по порядку величины примем $\mu=1, \mu_{0}=$ $=4 \pi \cdot 10^{-7} \mathrm{H} / \mathrm{m}, l=0.03 \mathrm{~m}, n=5000 \mathrm{~m}^{-1}, r=0.0005 \mathrm{~m}$, $c=3 \cdot 10^{8} \mathrm{~m} / \mathrm{s}, \omega=3 \cdot 10^{12} \mathrm{~Hz}$.

Столь высокое значение частоты взято, потому, что речь идет о микроволновом излучении с длиной волны $\lambda \sim 1 \mathrm{~mm}$. Амплитудное значение напряженности магнитного поля в электромагнитной волне от ШМ $H_{0}$ будем считать неизвестной величиной и оценим ее, принимая, что $\varepsilon_{\text {ind }}$ достаточно велика, чтобы вызвать свечение нити накаливания лампочки.

4. Сопротивление электролампочки $R$ в рабочем состоянии примем равным 500. На самом деле сопротивление нити накаливания при комнатной температуре примерно в 12-15 раз меньше, но по мере разогрева нити ее сопротивление увеличивается, и в рабочем режиме оно достигает нескольких сотен Ом. Ток, текущий через нить накаливания при этом уменьшается, и в рабочем режиме составляет десятые доли ампера. Для оценок по порядку величины примем, что $i \sim 0.1 \mathrm{~A}$.

Тогда получим

$$
\varepsilon_{\text {ind }}=i \cdot R \sim 0.1 \mathrm{~A} \cdot 500 \Omega \sim 50 \mathrm{~V} .
$$

Из (4) можно теперь найти амплитудное значение напряженности магнитного поля $H_{0}$, принимая, что синус в (3) равен единице:

$$
H_{0}=\frac{\varepsilon_{\text {ind }}}{\omega \pi r^{2} n \mu \mu_{0} l} \sim 0.05 \mathrm{~A} / \mathrm{m} .
$$

Объемная плотность энергии $w$ в электромагнитной волне определяется известными выражениями

$$
w=\varepsilon \varepsilon_{0} E^{2}=\mu \mu_{0} H^{2}=\sqrt{\varepsilon \varepsilon_{0} \mu \mu_{0}} E H .
$$

Используя удобную в данном случае формулу, получим плотность энергии в электромагнитной волне в виде

$$
w=\mu \mu_{0} H^{2} \equiv \mu \mu_{0} H_{0}^{2} \cos ^{2}\left[-\omega\left(t-\frac{x}{c}\right)\right] .
$$

Вектор плотности потока электромагнитной волны (вектор Умова-Пойнтинга) равен произведению w на вектор фазовой скорости волны $\mathbf{v}$, причем $\mathbf{v}=c / \sqrt{\varepsilon \mu}$, где $\varepsilon-$ диэлектрическая проницаемость:

$$
\mathbf{P}=\mathbf{w} \cdot \mathbf{v}
$$

Интенсивность $I$ электромагнитной волны равна модулю среднего значения вектора Умова-Пойнтинга

$$
I=|\langle P\rangle| \equiv\langle w\rangle \cdot v
$$

Здесь $\langle w\rangle$ - среднее значение объемной плотности энергии, равное

$$
\langle w\rangle=\mu \mu_{0} H_{0}^{2} \frac{2}{T} \int_{0}^{T / 2} \cos ^{2}\left[-\omega\left(t-\frac{x}{c}\right) \partial t\right]=\frac{1}{2} \mu \mu_{0} H_{0}^{2} .
$$

В итоге интенсивность излучения ШМ, если принять $\varepsilon=1, v \approx c$, равна по порядку величины

$$
I \approx \frac{1}{2} \mu \mu_{0} H_{0}^{2} c \sim 0.1 \mathrm{~W} .
$$

Таким образом, оценочное значение интенсивности радиоизлучения ШМ вблизи ее поверхности получилось весьма значительным.

5. Следует отдавать себе отчет в том, что все принятые выше для проведения оценки по порядку величины значения физических величин достаточно близки к реальным, но весьма условны. Тем не менее полученная оценка правдоподобна с энергетической точки зрения: энергия ШМ, идущая на излучение электромагнитных волн за $\sim 100$ s ее существования, составит лишь незначительную долю ее полной энергии, если исходить из оценки таковой в $\sim 10^{5} \mathrm{~J}$, полученной для минимальной энергии взрыва ШМ радиусом $\approx 0.25 \mathrm{~m}$ по разбросу кусков дерева, поврежденного этим взрывом (см., фотографию на задней обложке [12] или [14]).

В [29] приводится оценка полной энергии ШМ по последствиям расщепления бревна на щепки в $\sim 10^{8} \mathrm{~J}$. Автор [29] просто исправил расчетную ошибку в оценке [30]. Кроме того, автор [29] привел оценки энергии ШМ по произведенным ею разрушениям (со взрывом) в публикациях: [3], стр. 68; [5], стр. 102, 107; [11], стр. 59; [31], стр. 84; [32], стр. 108. В этих оценках нижний предел энергии ШМ был $\sim 4 \cdot 10^{6} \mathrm{~J}$, а верхний $\sim 10^{9} \mathrm{~J}$. Так что мы в своих оценках исходим из весьма умеренных данных

6. Говоря о физическом механизме электромагнитного излучения ШМ, можно предположить, что она излучает подобно тому, как излучает заряженная капля при осцилляциях своей поверхности [33]. Однако оценки интенсивности такого излучения дают весьма малую величину как для квадрупольного, так и для дипольного типов излучения [34], недостаточную для возбуждения свечения электролампочки. Вместе с тем следует отметить, что в [33] интенсивность излучения оценивается на расстоянии порядка километра от излучающего объекта, а для ШМ необходима оценка интенсивности вблизи ее поверхности. И тем не менее интенсивность излучения, связанного с колебаниями поверхности такого объекта, будет весьма малой даже с учетом падения величины интенсивности с расстоянием обратно пропорционально квадрату последнего. 
Достаточно очевидно, что физический механизм электромагнитного излучения ШМ должен быть связан с ускоренным движением внутри нее электрических зарядов. Что же можно сказать о структуре вещества ШМ? Что видят очевидцы внутри ШМ, когда она проходит на малом расстоянии от них? Примерно 13\% очевидцев, видевших ШМ с близкого расстояния [12], сообщают о наличии движущейся внутренней структуры ШМ. Они упоминают о хаотически движущихся светящихся точках, о переплетенных светящихся линиях, о движущихся в объеме ШМ маленьких светящихся шариках [12]. Некоторые сравнивают видимую структуру ШМ с „рыхлым, слабо намотанным клубком шерстяных нитей“.

Можно предположить, что за переплетенные светящиеся линии в силу инерционности зрительного восприятия могут приниматься следы быстро движущихся светящихся точек. Такие светящиеся точки движутся по криволинейным траекториям, а следовательно, с ускорением. Не задаваясь вопросом о природе светящихся точек, предположим, что они несут электрические заряды и могут быть источником электромагнитного излучения.

Согласно [27], ШМ имеет нескомпенсированный электрический заряд $Q_{b l}$ порядка нескольких микрокулон. Этот заряд может быть распределен между описанными выше движущимися светящимися точками.

В дипольном приближении интенсивность излучения одного движущегося с ускорением $а$ заряда на расстояниях, много больших длины волны (порядка километра), в системе СИ можно записать в виде [35, стр. 436]:

$$
I=\frac{q^{2} a^{2}}{6 \pi \varepsilon_{0} c^{3}},
$$

где $\varepsilon_{0}$ - электрическая постоянная, $\varepsilon_{0} \approx 8.85 \cdot 10^{-12} \mathrm{~F} / \mathrm{m}$.

Чтобы интенсивность излучения, рассчитываемую по формуле (5), можно было сравнить с найденной интенсивностью излучения у поверхности ШМ $I \sim 0.1 \mathrm{~W}$, последнюю нужно умножить на коэффициент $X$, учитывающий ослабление напряженности поля в волне с расстоянием. Численное значение этого коэффициента определится выражением $X \sim\left(L_{2} / L_{1}\right)^{2}$, если принять, что (5) дает интенсивность поля в волне на расстоянии $L_{1}$, а расстояние от ШМ до лампочки $L_{2}$. Принимая $L_{1} \sim 1000 \mathrm{~m}, L_{2} \sim 0.1 \mathrm{~m}$, получим $X \sim 10^{-8}$.

Чтобы заряд $Q_{b l} \sim 6 \cdot 10^{-6} \mathrm{C}$ [27] при ускоренном движении излучал с интенсивностью $I \sim 0.1 \mathrm{~W}$ непосредственно рядом с ШМ, он должен иметь ускорение

$$
a=\frac{1}{2} \sqrt{6 \pi \varepsilon_{0} c^{3} \cdot I \cdot X} \sim 10^{8} \frac{\mathrm{m}}{\mathrm{s}^{2}} .
$$

Это очень большое ускорение, особенно если принять во внимание характерный линейный размер ШМ $R_{b l} \sim 0.1 \mathrm{~m}$.

Учтем теперь, что это ускорение будет центростремительным: $a=\left(V^{2} / R_{b l}\right)$. Из этого соотношения можно найти скорость движения светящихся точек: $V \sim \sqrt{a R_{b l}}$ или, переходя к цифрам, получим $V \sim 3000 \mathrm{~m} / \mathrm{s}$.

Человеческий глаз не замечает явления, происходящие быстрее чем за $0.1 \mathrm{~s}$ [36], поэтому если светящаяся точка движется с большой скоростью, то человек воспримет ее как светящуюся линию. Разделив длину половины окружности на скорость движения заряда, мы сможем определить время наблюдения $\tau$ на обращенной к очевидцу половинке ШМ. Если $<0.1 \mathrm{~s}$, то человеческий глаз воспримет траекторию движения как светящуюся линию, если $\tau>0.1 \mathrm{~s}$, то глаз зафиксирует движение светящейся точки. Именно эти варианты ответов респондентов на вопрос о наличии видимой структуры ШМ и регистрируются в реальности.

Проведенная оценка ускорения движения светящихся частиц внутри ШМ существенно зависит от величины нескомпенсированного заряда ШМ, который был принят, согласно строгим расчетам [27], равным $Q_{b l} \sim 6 \cdot 10^{-6} \mathrm{C}$ при радиусе $R_{b l} \sim 0.15 \mathrm{~m}$. Следует отметить, что более двадцати лет назад в [37] из общефизических соображений для заряда ШМ получена аналогичная оценка.

Заметим, что полученное значение ускорения можно значительно уменьшить, если учесть, что кроме нескомпенсированного заряда, ШМ может нести и скомпенсированные заряды двух противоположных знаков, индуцированные приземным электрическим полем.

Величина заряда, индуцированного в электропроводной сфере радиуса $R$ внешним полем $E_{0}$, определится соотношением $Q \sim E_{0} R^{2}$. Примем для оценки по порядку величины, что $E_{0}-$ напряженность приземного электрического поля в грозовую погоду, составляет $\sim 1000 \mathrm{~V} / \mathrm{m}[38]$, а радиус ШМ $R_{b l}=0.15 \mathrm{~m}$. Тогда получим, что величина индуцированного в сфере (читай в ШМ) заряда будет $Q \sim 20$ С. А именно общий заряд, участвующий в создании электромагнитного излучения, будет на семь порядков больше. Тогда, согласно (5), для величины ускорения движения частиц в ШМ получим оценку на семь порядков величины меньше, а оценка скорости движения светящихся точек в объеме ШМ даст $V \sim 1 \mathrm{~m} / \mathrm{s}$. При характерном радиусе ШМ $R_{b l} \sim 0.1 \mathrm{~m}$ такая скорость движения попадает на границу, разделяющую описанные выше варианты визуального восприятия структуры ШМ: сплошные светящиеся линии или быстро движущиеся светящиеся точки.

\section{Заключение}

В физически непротиворечивых общефизических оценках в согласии с данными наблюдений ШМ в естественных условиях оценена интенсивность электромагнитного микроволнового излучения ШМ вблизи ее поверхности $\sim 0.1 \mathrm{~W}$. Электромагнитное излучение такой мощности может быть вызвано движением в объеме ШМ по криволинейным траекториям заряженных частиц. 


\section{Список литературы}

[1] Араго Ф. Гром и молния. СПб.: Изд-во Императорской $\mathrm{AH}, 1859.424 \mathrm{c}$.

[2] Brand $W$. Der Kugelblitz. Probleme der Kosmischen Physik. Hamburg: H. Grand, 1923. $170 \mathrm{p}$

[3] Леонов Р.А. Загадка шаровой молнии. М.: Наука, 1965. $75 \mathrm{c}$.

[4] Имянитов И.М., Тихий Д.Я. За гранью закона. Л.: Гидрометеоиздат, $1967.141 \mathrm{c}$.

[5] Сингер С. Природа шаровой молнии. / Пер. с англ. М.: Мир, 1973. 239 с.

[6] Стаханов И.П. Физическая природа шаровой молнии. М.: Атомиздат, 1979. $242 \mathrm{c.}$

[7] Смирнов Б.М. Проблема шаровой молнии. М.: Наука, 1988. $209 \mathrm{c}$

[8] McNally J.R. Jr. Second Annual Meeting of the Division of Plasma Physics of the American Physical Society. Gatlinburg, 1960. N 2-5. P. 1-25.

[9] Rayle W.D. Rept. NASA., T.N.O. 1966. P. 3188.

[10] Charman W.N. // Phys. Rep. 1979. Vol. 54. N 4. P. 261-306.

[11] Барри Джс. Шаровая молния и четочная молния. / Пер. с англ. М.: Мир, 1983. 285 с.

[12] Григорьев А.И. Шаровая молния. Ярославль: Изд-во ЯрГУ, 2010. $200 \mathrm{c}$

[13] Science of Ball Lightning. Ed. Y.H. Ohtsuki. Singapure: World Scientific, 1989. 340 p.

[14] Долгоживущие плазменные образования и малоизученные формы естественных электрических разрядов в атмосфере. Матер. VIII Междунар. конф. 4-8 июня 2009г. Ярославль. 2009. 208 с.

[15] Kirko D.L. // Low Temperature Physics. 2015. Vol. 41. N 4. P. 303-307.

[16] Пирозерский А.Л., Михайловский В.Ю. и др. // Электрогидродинамика проводящей жидкости. Долгоживущие плазменные образования и малоизученные формы естественных электрических разрядов в атмосфере. Матер. ХІ Междунар. конф. 04-07 июля 2015 г. Ярославль. C. $105-111$.

[17] Кирко Д.Л., Савелов А.С. // ТВТ. 2015. Т. 53. № 6. С. 953956.

[18] Емелин С.Е., Семенов В.С. и др. // ЖТФ. 1997. Т. 67. Вып. 3. С. 19-28.

[19] Шабанов Г.Д. // Письма в ЖТФ. 2002. Т. 28. Вып. 4. С. 8186.

[20] Фуров Л.В. // ЖТФ. 2005. Т. 75. Вып. 3. С. 96-99.

[21] Егоров А.И., Степанов С.И. // ЖТФ. 2006. Т. 78. Вып. 6. C. $15-19$.

[22] Шабанов Г.Д., Крившич А.Г., Жеребцов О.М., Гаврилов Г.Е. // Электрогидродинамика проводящей жидкости. Долгоживущие плазменные образования и малоизученные формы естественных электрических разрядов в атмосфеpe. Матер. Х Междунар. конф. 4-8 июля 2013 г. Ярославль. С. 214-225.

[23] Стаханов И.П. // ЖТФ. 1974. Т. 44. Вып. 7. С. 1373-1379.

[24] Колосовский О.А. // ЖТФ. 1999. Т. 69. Вып. 10. С. 856-858.

[25] Бойченко А.М. // ЖТФ. 1974. Т. 44. Вып. 7. С. 131-134.

[26] Bychkov V.L., Nikitin A.И. et al. // J. Atmospheric and SolarTerrestrial Physics. 2016. N 150. P. 69-76.

[27] Григорьев А.И., Петрушов Н.А., Ширяева С.О. // ЖТФ. 2016. Т. 86. Вып. 9. С. 39-47. [Tech. Phys. 2016. Vol. 61. N 9. P. 1319-1327.]
[28] Petrushov N.A., Grigor'ev A.I., Shiryaeva S.O. // Surfa. Engineer. Appl. Electrochem. 2017. Vol. 53. N 2. P. 144-149.

[29] Козлов Б.Н. // ДАН СССР. 1978. Т. 238. № 1. С. 61-66.

[30] Zimmerman P. // Nature. 1970. Vol. 228. N 28. P. 853.

[31] Телетов Г.С. // Природа. 1960. № 9. С. 84.

[32] Lewis H.W. // Scientific American. 1963. Vol. 208. N 3. P. 106-116.

[33] Калечии В.И., Нахутин И.Е., Полуэктов П.П. // ДАН CССР. 1982. T. 262. № 6. С. 1344-1347.

[34] Григорьев А.И., Ширяева С.О. // ЖТФ. 2016. Т. 86. Вып. 12. С. 129-134. [Tech. Phys. 2016. Vol. 61. N 12. P. 1895-1900.]

[35] Ахиезер А.И., Ахиезер И.А. Электромагнетизм и электромагнитные волны. М.: Высш. шк., 1985. 504 с.

[36] Луизов А.В. Глаз и свет. Л.: Энергоатомиздат, 1983. 140 с.

[37] Смирнов Б.М. // УФН. 1992. Т. 162. № 8. С. 43-81.

[38] Матвеев Л.Т. Курс общей метеорологии. Физика атмосферы. Л.: Гидрометеоиздат, 1984. 751 с. 